\title{
PARAFAC: Adjustment for modeling consumer study covering probiotic and conventional yogurt
}

\author{
Adriano G. Cruz ${ }^{\text {a, } *}$, Rafael S. Cadena ${ }^{a}$, José A.F. Faria ${ }^{a}$, Helena M.A. Bolini ${ }^{a}$, Clecio Dantas ${ }^{b}$, \\ Márcia M.C. Ferreira ${ }^{\mathrm{b}}$, Rosires Deliza ${ }^{\mathrm{c}}$ \\ a Universidade Estadual de Campinas (UNICAMP), Faculdade de Engenharia de Alimentos (FEA), Cidade Universitária Zeferino Vaz CEP: $13083-862$ - Campinas - São Paulo - Brasil \\ b Universidade Estadual de Campinas (UNICAMP), Instituto de Química (IQM). Cidade Universitária CEP: 13083-970 - Campinas, São Paulo - Brasil

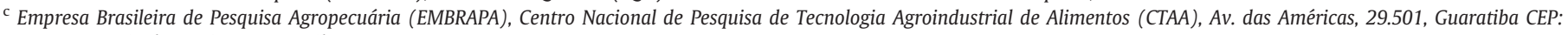 \\ 23020-470 - Rio de Janeiro, RJ - Brasil
}

\section{A R T I C L E I N F O}

\section{Article history:}

Received 27 July 2011

Accepted 18 September 2011

\section{Keywords:}

Conventional yogurt

Probiotic yogurt

Consumer study

PCA

PARAFAC

\begin{abstract}
A B S T R A C T
The feasibility of the parallel factors model (PARAFAC) as a modeling tool for consumer sensory acceptance data of complex food matrices was investigated. The attributes of aroma, flavor, taste, texture, and overall liking of probiotic and conventional yogurts were evaluated by 120 consumers using a 9-point hedonic hybrid scale. Six yogurt samples were used: three were prototypes supplemented with a glucose oxidase/glucose system, which is a potential oxygen scavenging system, and the other three were commercial yogurts. The yogurts supplemented with the glucose oxidase/glucose system presented similar sensory acceptance towards commercial probiotic and conventional yogurts ( $p>0.05)$, besides having garnered lower mean scores for the sensory attributes. Appearance and overall liking were the most significant attributes for the first two components of the PARAFAC model. Finally, the advantage of using this method to explore the intrinsic nature of consumer sensory data is discussed and compared to principal component analysis (PCA).
\end{abstract}

(C) 2011 Elsevier Ltd. All rights reserved.

\section{Introduction}

The constant changing of current consumer needs and the huge amount of options in the marketplace together with the need for making him/her keeping purchasing the product have made the clear understanding of the sensory aspects a compulsory issue (Tuorilla \& Monteleone, 2009). Among these sensory aspects, the overall liking is essential and determines the repeated consumption and purchase of the product (Lim, 2011).

For functional foods, such as probiotic yogurts, the sensory properties of the products are essential, once they should display similar sensory acceptance/characteristics towards similar conventional foods. This aspect needs to be taken into consideration in functional food development once the supplementation of yogurt with probiotic bacteria can proportionate a positive or negative effect on the sensory attributes (texture, aroma, and flavor) (Cruz, Cadena et al., 2010; Cruz, Faria et al., 2010; Cruz, Walter et al., 2010). Other crucial topic to be pointed out is that most researchers focus the performance of technological options related to the survival of probiotic (Marafon, Sumi, Alcântara, Tamime, \& Oliveira, 2011; Oliveira, Perego, Oliveira, \& Converti, 2011; Isik, Boyacioglu, Capanoglu, \& Erdil, 2011), minimization of the oxidative stress (Li, Chen, Ruan, Zhu \& He, 2010; Yonezawa et al., 2010; Ebel,

\footnotetext{
* Corresponding author. Tel.: +55 1932514016.

E-mail address: adriano@fea.unicamp.br (A.G. Cruz).
}

Martin, Le, Gervai, \& Cachon, 2011; Martin et al., 2011) and microencapsulation (Ding \& Shah, 2009), without mentioning the acceptance of the final products compared to commercial products.

To improve this complex food matrix, it is very important to understand the consumer's preferences and expectations in order to ensure the product's acceptance. In this regard, the modeling of consumer's data is an important and essential factor to be considered. Parallel Factor Analysis (PARAFAC) is a chemometric method employed for decomposition of higher order data, and therefore this technique has been widely used in sensory analysis. By using PARAFAC, it is possible to provide valuable information concerning agreement within the trained panelists with respect to the product's sensory attributes, as well the performance of a certain attribute in relation to a particular panelist in modeling sensory data (Brockhoff, Hirst \& Naes, 1996).

The PARAFAC method has been explored in the quantitative descriptive analysis of cream cheese (Bro, Qannari, Kiers, Naes, \& Frost, 2008) and of 'aceto balsâmico tradizionale di Modena', a typical Italian product protected by the denomination of origin (Cocchi et al., 2005). Another application of this method was reported in the modeling of the data from dynamic sensory tests, such as the temporal perception of flavor in chewing gum (Ovejero-López, Bro, \& Bred, 2005).

Recently, the potential use of PARAFAC as a tool to model consumer sensory acceptance data has been demonstrated. This multivariate approach provided a suitable explanation towards the data variability of consumer preferences of beef burger and herb cake (Nunes, Pinheiro, \& Bastos, 2011). 
However, it is important to evaluate the performance of PARAFAC in complex food matrices - such as yogurts - products that contain stabilizers and thickeners as part of their formulation. In this regard, the feasibility of using this chemometric tool to investigate the sensory performance of conventional and probiotic yogurts seems promising, once relevant results can be obtained towards these groups of products, which belong to the same food product category, but sometimes are perceived differently by consumers. (Heydari, Mortazavian, Ehsani, Mohammadifar \& Ezzatpanah, 2011; Majchrazak, Lahm, \& Durrschmid, 2010; Allgeyer, Miller \& Lee, 2010).

This research aimed at evaluating the potential of PARAFAC as a modeling tool of acceptance data of complex food matrices belonging to different subgroups of a same category: probiotic and conventional non-flavored yogurts. For comparative purposes, principal component analysis (PCA) was also performed on the consumer acceptance data.

\section{Material and methods}

\subsection{Sampling}

Six different non-flavored yogurts were used in this study. Three were commercial yogurts $\left(C_{1}, C_{2}\right.$ and $\left.C P\right)$ from well-known brands available in the Brazilian marketplace. Two of them were conventional $\left(C_{1}\right.$ and $C_{2}$, similar nutrient composition as it mentioned on the label), whilst the third one was a probiotic yogurt (CP) supplemented with Bifidobacteirum animalis DN $173010 \mathrm{~W}$. The other three $\left(\mathrm{P}_{1}, \mathrm{P}_{2}\right.$ and $\mathrm{P}_{3}$ ) were probiotic yogurts elaborated with the following cultures: Streptococcus thermophilus TA 040, Lactobacillus delbrueckii ssp. bulgaricus LB 340 with Bifidobacterium longum BL 05 and Lactobacillus acidophilus LA 14 (Danisco, São Paulo, Brazil). The probiotic yogurts were also supplemented with $62.32 \mathrm{ppm}$ glucose oxidase and 4.35 ppm glucose, a potential oxygen scavenging system that guarantees the survival of probiotic bacteria. The products were packed into different plastic packaging systems with oxygen permeability values of $0.09,0.20$ and $0.39 \mathrm{~cm}^{3} /$ package/day, respectively. Details of the manufacture are published elsewhere (Cruz, Faria et al., 2010; Cruz et al., 2011).

\subsection{Consumer test}

One hundred and twenty yogurt consumers (Drake, 2007) were recruited at random at the State University of Campinas to take part in the study. The criterion for selection was the regular consumption of yogurt. The consumers were asked to evaluate the yogurts with respect to the degree of liking of appearance, aroma, taste, texture and overall acceptance, using a 9-point hybrid hedonic scale $(1=$ disliked immensely, 9 = liked immensely) (Villanueva \& Da Silva, 2009). Between tasting each sample, the participants were requested to eat a cream cracker biscuit and drink some water. The first-order and carry-over effects were balanced using a specific design, where each consumer evaluated all the yogurt samples (MacFie, Bratchell, Greenhoff, \& Vallis, 1989). All analyses were carried out in individual booths, with controlled temperature and humidity conditions. The test was duly approved by the Ethic Committee of the Faculty of Medical Science of UNICAMP, under the protocol number 295/2008. Moreover, a written consent was given by all volunteers before starting the test.

\subsection{Univariate statistics}

The consumer test data were initially submitted to the Levene's test to verify the homogeneity of variances. An analysis of variance was carried out in the second step, where consumers and samples were the sources of variation, followed by Tukey's test (5\% significance level). All these analyses were carried out using the Statistica
7.1 software (StatSoft TM, Tulsa, OK, USA), at the 95\% significance level.

\subsection{PCA and PARAFAC}

PCA and PARAFAC are bi- and multi-linear decomposition methods, respectively, which decompose the array into sets of scores and loadings that describe the data in a more condensed form than the original data array. They assume the existence of a latent structure in each mode of the data sets, which allows one to compact related descriptors and describe the variance within each mode by a reduced number of factors, which facilitates the interpretation of relevant information (Bro, 1997).

One characteristic of the consumer sensory acceptance data is the lack of a theory which describes it, once people have different preferences (Lim, 2011). In this regard, the application of chemometric methods is an elegant way of exploring data of this nature.

In general, consumer's data have the following form: $k$ consumers judge $I$ products with respect to $J$ attributes. They can be arranged in two ways (Fig. 1): i) a matrix $\mathbf{X}_{\mathrm{m}}$ with the dimensions (I-samples $x$ $J$-attributes) obtained from the average of the scorings per consumer; ii) a three-way array $\mathbf{X}$ with three dimensions (I-samples $x$ $J$-attributes $\times K$-consumers).

In the first way, a two-dimensional array is explored by PCA through an average configuration matrix, where the average information about the consumers is considered. In the second, a threedimensional array is evaluated by PARAFAC and individual consumer's information is taken into consideration.

In the PARAFAC method, a trilinear model is found to minimize the sum of squares of the residues, $e_{i j k}$, according to Eq. (1).

$x_{i j k}=\sum_{f=1}^{F} a_{i f} b_{j f} c_{k f}+e_{i j k}$

The most important step when the PARAFAC method is applied consists of estimating the number of components or latent variables (F) in the data set (see Eq. (1)). Several criteria can be used to determine this parameter, such as the explained variance and core consistency diagnostic (CORCONDIA) (Bro \& Kiers, 2003). CORCONDIA values are always less than or equal to $100 \%$ and may also be negative. A core consistency close to $100 \%$ implies an appropriate model. A core consistency in the neighborhood of $50 \%$ would mean a problematic model. A core consistency close to zero or even negative implies an invalid model (Bro \& Kiers, 2003).

In the present study, PARAFAC models with different numbers of factors were calculated and the criteria CORCONDIA and explained variance were used to determine the appropriate number of components. This number was assessed through of the model with the highest number of components, the greatest explained variance and a valid value for CORCONDIA, close to $100 \%$.

The PARAFAC algorithm used in this work was from the N-way Toolbox (Andersson \& Bro, 2000), a program written in MATLAB code and downloaded at http://www.models.life.ku.dk/source/ nwaytoolbox/.


Fig. 1. Data array arrangement of I products towards the J attributes for K consumers. 


\section{Results and discussion}

\subsection{Consumer test}

Table 1 shows the sensory results obtained with consumers for the probiotic and conventional yogurts. Besides the fact that the yogurts supplemented with $B$. longum and the glucose oxidase/glucose system (P1, P2 and P3) presented lower mean scored for the sensory attributes, these samples garnered similar $(\mathrm{p}>0.05)$ values compared to the commercial probiotic yogurt $(\mathrm{CP})$ and also with respect to the conventional yogurts ( $\mathrm{C} 1$ and $\mathrm{C} 2$ ).

For aroma and texture, the mean scores ranged from 5.0 (neither liked nor disliked) to 6.0 (liked slightly), whilst for taste, the mean scores were between 4.0 (disliked slightly) and 5.0 (neither liked nor disliked). Again, the commercial yogurts presented a higher sensory performance towards the prototypes $(p<0.05)$. As expected, the mean values for taste and overall liking were between 5.0 (neither liked nor disliked) and 6.0 (liked slightly).

Although the purchase of functional foods, such as probiotic yogurts is influenced by different aspects, the intrinsic (e.g. color and flavor) and extrinsic (e.g. price, origin, health claim, quality stamp, production and nutritional information) factors are also used to create expectations about the product's quality attributes (Menezes, Deliza, Cham, \& Guinard, 2011; Ngapo, Fortin, Aalhus, \& Martin, 2010). It has also been shown that the brand, price and health claims affect consumer's choice in a highly significant way (Ares, Giménez, \& Deliza, 2010) as well as the nutritional and pleasure aspects (Ares, Giménez, \& Gambaro, 2010). However, the sensory aspect remains as determinant for their acceptance by consumers (Bayarri, Carbonell, Barrios, \& Costell, 2010).

Overall, our findings suggest that the addition of an oxygen scavenging system composed of glucose oxidase/glucose was compatible, from the sensory point of view, once a similar acceptance between prototypes and the commercial yogurts was reported.

\subsection{PARAFAC modeling}

The data were organized in a three-way array $\mathbf{X}$ with dimensions of $6 \times 5 \times 120$ referring to samples $\times$ attributes $\times$ consumers (Fig. 1 ). No pre-treatment was used in this modeling.

Analyzing the performance of the calculated PARAFAC models, one can affirm that the model with 2 components was adequate, since the values for CORCONDIA (100\%) and the explained variance (95.02\%) confirmed the model's validity (Table 2 ).

Fig. 2 shows the graphs of the scores and loadings referring to the modes described by the matrices $\mathbf{A}, \mathbf{B}$ and $\mathbf{C}$ for the first two components. In the graph of scores, Fig. 2a, sample P1 shows highly distinct acceptance as compared to the other samples, mainly with respect to the degree of liking of taste, overall liking and appearance. This result can be related to lower permeability oxygen rate of the package, which improved the viability of $B$. longum (Cruz, Faria, et al., 2010). This anaerobic environment proportionated better conditions for the growth of the probiotic bacteria, resulting at increased

Table 1

Consumer mean acceptance of probiotic and conventional yogurts *

\begin{tabular}{llllll}
\hline Yogurts & Appearance & Aroma & Taste & Texture & Overall liking \\
\hline P1 & $5.87^{\mathrm{b}}$ & $5.86^{\mathrm{b}}$ & $4.94^{\mathrm{a}}$ & $5.15^{\mathrm{b}}$ & $5.06^{\mathrm{a}}$ \\
P2 & $6.84^{\mathrm{a}}$ & $6.07^{\mathrm{a}}$ & $4.81^{\mathrm{a}}$ & $6.04^{\mathrm{a}}$ & $5.22^{\mathrm{a}}$ \\
P3 & $6.72^{\mathrm{a}}$ & $6.34^{\mathrm{a}}$ & $4.77^{\mathrm{a}}$ & $6.39^{\mathrm{a}}$ & $5.47^{\mathrm{a}}$ \\
C1 & $7.02^{\mathrm{a}}$ & $6.02^{\mathrm{a}}$ & $4.38^{\mathrm{b}}$ & $6.07^{\mathrm{a}}$ & $5.56^{\mathrm{a}}$ \\
C2 & $6.59^{\mathrm{a}}$ & $6.22^{\mathrm{a}}$ & $5.45^{\mathrm{a}}$ & $6.05^{\mathrm{a}}$ & $5.71^{\mathrm{a}}$ \\
CP & $6.74^{\mathrm{a}}$ & $6.28^{\mathrm{a}}$ & $5.07^{\mathrm{a}}$ & $5.93^{\mathrm{a}}$ & $5.52^{\mathrm{a}}$ \\
\hline
\end{tabular}

*Mean data from 100 consumer and based on a 9-point hedonic scale $(1=$ dislike extremely. $5=$ neither like nor dislike. $9=$ like extremely). Different lowercase in the same column in indicate presence of statistical difference $(\mathrm{p}<0.05)$ among treatments (yogurts).
Table 2

PARAFAC models performance.

\begin{tabular}{lllccc}
\hline & 1 & 2 & 3 & 4 & 5 \\
\hline CORCONDIA (\%) & 100.0 & 100.0 & 5.140 & 0.644 & 0.141 \\
\% Var. explained & 93.95 & 95.02 & 95.83 & 96.42 & 96.88 \\
\hline
\end{tabular}

production of acidic metabolites such as acetic and lactic acids, contributing for a lower overall acceptance of this sample.

Samples P2 and CP were similarly liked by participants. The loadings plot for the two first dimensions of the second mode, that is, the mode of the attributes, is presented in Fig. 2b. For the first component, the appearance was the most important attribute in explaining the disposition of the samples, whilst for the second component, the


Fig. 2. Biplot of A, B and $\mathbf{C}$ matrices obtained by PARAFAC. a) Mode 1, score plot (samples); b) Mode 2, loadings plot (sensory attributes) and c) Mode 3, loadings plot (consumers). 
overall liking and flavor presented the greatest loadings, confirming the importance of these attributes on the product's evaluation. These results corroborate those described in the literature for chocolate (Palazzo \& Bolini, 2009), carrot cupcakes (Villanueva, Doris, \& Trindade, 2010) and probiotic cheese (Gomes, Braga et al., 2011) and low sodium cheese (Gomes, Cruz et al., 2011).

In addition, a correlation between the degree of liking of aroma and texture was visualized, which may be due to the ingredients used (thickening agent) and to the parameters (intensity of the mechanical treatment applied to break the gel) adopted during the processing of the yogurts (Kora, Latrille, Souchon, \& Martin, 2003; Saint-Eve, Kora, \& Martin, 2004).

Overall, these findings suggest that these attributes (aroma and texture) were perceived in a similar way by the consumers.

Fig. 2c shows the loadings plot for the two first factors of the third mode, i.e., the consumer's information. Here one can infer that consumers were more homogeneous in evaluating the attributes of the first component, and highly heterogeneous in evaluating, principally, the appearance and overall liking of the second component. This information is not new, as the divergences in the scores attributed by some consumers reflected the different modes of liking of the products.

\subsection{PCA modeling}

Principal components analysis (PCA) was applied to the matrix $\mathbf{X}_{\mathbf{m}}$ with dimensions of $(6 \times 5)$, obtained by calculating the average consumer assessments for acceptance of the yogurts. The data were mean-centered and the result of this analysis was presented using a biplot graph (Fig. 3).

Analyzing the first two principal components, 95.13\% of the variance in the data was explained by the model, allowing the evaluation of the samples that were best linked by the average consumers. For PC1, sample P1 received the lowest mean scores for texture and appearance. Samples C1 and P2 garnered the highest scores for appearance, and sample P3 garnered the highest scores for texture and aroma. For PC2, sample $\mathrm{C} 1$ received the lowest scores for flavor and overall liking, whilst sample $\mathrm{C} 2$ presented the highest scores. In general, one could say that the texture and appearance were the principal attributes to explain approximately $61 \%$ of the variation in the data. Flavor and overall impression accounted for the remaining 34\% of the variability.

\subsection{PARAFAC and PCA modeling}

Some differences were observed between the results obtained considering the individual scoring (PARAFAC) and the result obtained considering a mean value for the scoring given by the consumers (PCA). The similar evaluation obtained between samples $\mathrm{P} 2$ and $\mathrm{CP}$, and between $\mathrm{C} 2$ and $\mathrm{P} 3$, as also the correlation between aroma and texture was not so evident in the analysis performed by PCA in comparison to the analysis performed by PARAFAC.

The degree of liking of texture was the greatest loading in the first component using PCA. On the other hand, using PARAFAC, this attribute was only the third most important. The overall liking presented the greatest loading in the second component obtained by PARAFAC, whereas the degree of liking of taste was the greatest loading in PCA.

From the sensory of view, the PARAFAC model presented a more informative graphic display compared to PCA, where the individual information of consumers was considered. This fact allowed us to assess how consumers used the scale.

\section{Conclusion}

The PARAFAC method seems to be a potential option to model sensory data. Its use provided intrinsic information of the consumer's perception about the prototypes and commercial probiotic/conventional yogurts.

According to this model, the prototypes and commercial products (P2-CP and P3-C2) presented similar sensory characteristics. This suggests that the packing system with adequate oxygen permeability rate is essential to a better sensory performance of the probiotic yogurts that contain glucose oxidase/glucose. Additionally, the supplementation with probiotic bacteria did not influence of the overall liking of the probiotic yogurts.

This study presents limitations. Firstly, we used a small number of samples and a panel composed by 120 yogurt consumers, a number that is not representative of the population; secondly, we did not collect the consumer's socio-demographic information, therefore it was not possible to explore the results in relation to gender, educational level, age, among other variables. However, our findings are valuable once they report several correlations among the sensory scores of a complex food matrix, such as yogurts, which is manufactured using

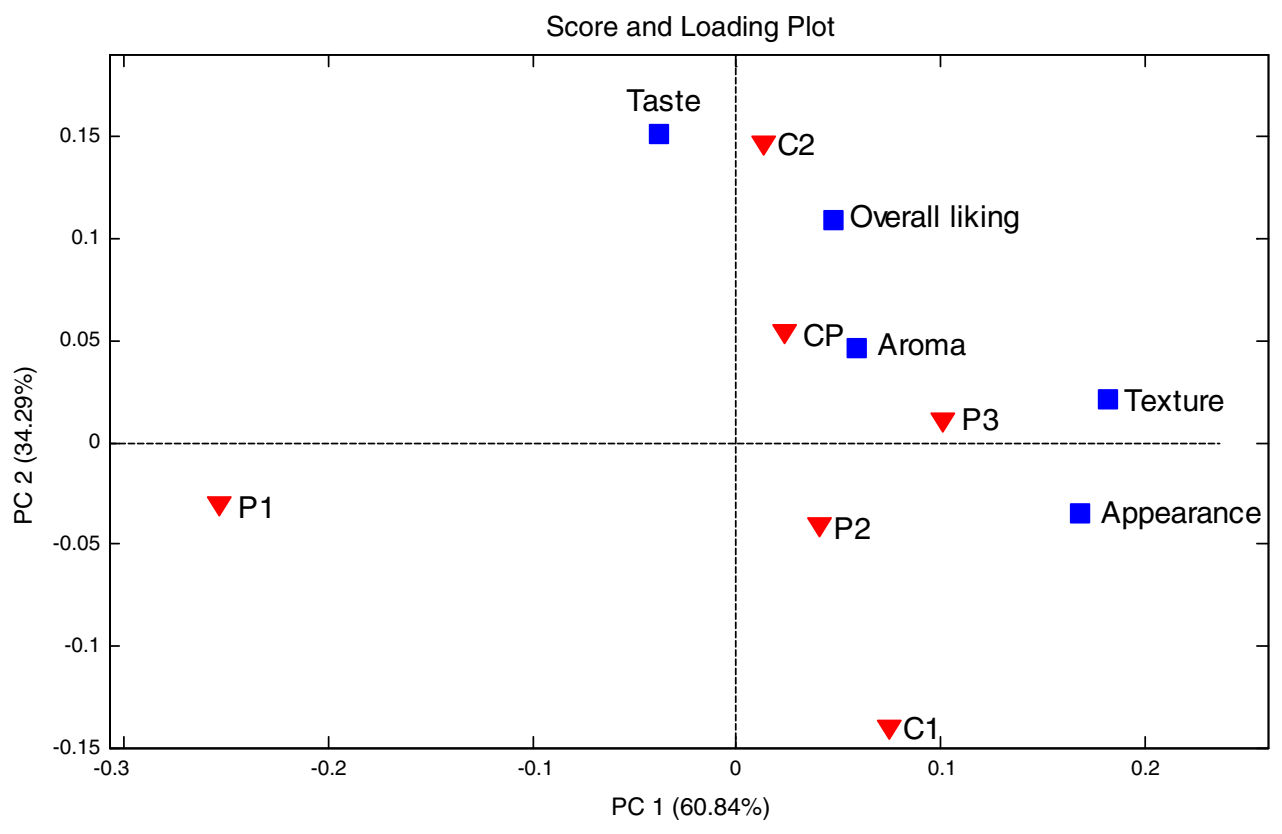

Fig. 3. Biplot of PCA analysis. 
different lactic cultures with different metabolism profile. In addition, it contains mixtures of stabilizers, thickeners, resulting in different formulations. Another important point it was possible to check the performance of prototypes towards similar commercial conventional/ probiotic products with established formulations.

Considering the complexity of the food matrix analyzed (yogurts) and the intrinsic and extrinsic factors assessed in the sensory evaluation of the probiotic and conventional yogurts, our results are useful and deserve consideration by the dairy industry. Further sensory studies covering other food matrices are needed to confirm the potential use of PARAFAC as an option to model consumer acceptance data.

\section{References}

Allgeyer, L. C., Miller, M. J., \& Lee, S. -Y. (2010). Sensory and microbiological quality of yogurt drinks with prebiotics and probiotics. Journal of Dairy Science, 93, 4471-4479.

Andersson, C. A., \& Bro, R. (2000). The N-way toolbox for MATLAB. Chemometrics and Intelligent Laboratory Systems, 52, 1-4.

Ares, G., Giménez, A., \& Deliza, R. (2010). Influence of three non-sensory factors on consumer choice of functional yogurts over regular ones. Food Quality and Preference, $21,361-367$.

Ares, G., Giménez, A., \& Gambaro, A. (2010). Understanding consumers' perception of conventional and functional yogurts using word association and hard laddering. Food Quality and Preference, 19, 636-643.

Bayarri, S., Carbonell, I., Barrios, E. X., \& Costell, E. (2010). Acceptability of yogurt and yogurt-like products: Influence of product information and consumer characteristics and preferences. Journal of Sensory Studies, 25, S171-S210.

Bro, R. (1997). PARAFAC: Tutorial and applications. Chemometrics and Inteligent Laboratory Systems, 38, 149-171.

Bro, R., \& Kiers, H. A. L. (2003). A new efficient method for determining the number of components in PARAFAC models. Journal of Chemometrics, 17, 274-286.

Bro, R., Qannari, E. M., Kiers, H. A. L., Naes, T., \& Frost, M. B. (2008). Multi-way models for sensory profiling data. Journal of Chemometrics, 22, 36-45.

Brockhoff, P. M., Hirst, D., \& Naes, T. (1996). Analysing individual profiles by three-way factor analysis. In T. Naes, \& E. Risvik (Eds.), Multivariate analysis of data in sensory science (pp. 307-342). New York: Elsevier.

Cocchi, M., Bro, R., Durante, C., Manzini, D., Marchetti, A., Saccani, F., et al. (2005). Analysis of sensory data of Aceto Balsamico Tradizionale di Modena (ABTM) of different ageing by application of PARAFAC models. Food Quality and Preference, 17, 419-428.

Cruz, A. G., Cadena, R. S., Faria, J. A. F., Oliveira, C. A. F., Cavalcanti, R. N., Bona, E., Bolini, H. M. A., \& Da Silva, M. A. A. P. (2011). Consumer acceptability and purchase intent of probiotic yoghurt with added glucose oxidase using sensometrics, artificial neural networks and logistic regression. International Journal of Dairy Technology, 64, 549-556.

Cruz, A. G., Cadena, R. S., Walter, E. H. M., Mortazavian, A., Granato, D., Faria, J. A. F., et al. (2010). Sensory analysis: Relevance for prebiotic, probiotic, and synbiotic product development. Comprehensive Reviews in Food Science and Food Safety, 9, 358-373.

Cruz, A. G., Faria, J. A. F., Walter, E. H. M., Andrade, R. R., Walter, E. H. M., Cavalcante, R. N. et al. (2010). Processing optimization of probiotic yogurt containing glucose oxidase using response surface methodology. Journal of Dairy Science, 93, 5059-5068.

Cruz, A. G., Walter, E. H. M., Cadena, R. S., Faria, J. A. F., Bolini, H. M. A., Pinheiro, H. P., et al. (2010). Survival analysis methodology to predict the shelf-life of probiotic flavored yogurt. Food Research International, 43, 1444-1448.

Ding, W. K., \& Shah, N. P. (2009). Effect of homogenization techniques on the reducing the size of microcapsules and the survival of probiotic bacteria therein. Journal of Food Science, 74, 231-236.

Drake, M. A. (2007). Sensory analysis of dairy foods. Journal of Dairy Science, 11, 4925-4937.

Ebel, B., Martin, F., Le, L. D. T., Gervai, P., \& Cachon, R. (2011). Use of gases to improve survival of Bifidobacterium bifidum by modifying redox potential in fermented milk. Journal of Dairy Science, 94, 2185-2191.
Gomes, A. A., Braga, S. P., Cruz, A. G., Cadena, R. S., Faria, J. A. F., Lollo, P. C., Amaya-Farfan, J., Carvalho, C. C., \& Bolini, H. M. A. (2011). Effect of the inoculation level of L. acidophilus in probiotic cheese on the physicochemical features and sensory performance towards commercial cheeses. Journal of Dairy Science, 94, 4777-7786.

Gomes, A. P., Cruz, A. G., Cadena, R. S., Faria, J. A. F., Celeguini, R. M. S., Bolini, H. M. A., \& Pollonio, M. A. R. (2011). Low sodium Minas fresh cheese manufacture: Effect of the partial replacement of $\mathrm{NaCl}$ with $\mathrm{KCl}$. Journal of Dairy Science, 94 27062011.

Heydari, S., Mortazavian, A. M., Ehsani, M. R., Mohammadifar, M. A., \& Ezzatpanah, H. (2011). Biochemical. Microbiological and Sensory charateristics of probiotic yogurt containing prebiotic compounds. Italian Journal of Food Science, 23, 153-163.

Isik, U., Boyacioglu, D., Capanoglu, E., \& Erdil, D. N. (2011). Frozen Yogurt with added inulin and isomalt. Journal of Dairy Science, 94, 1647-1656.

Kora, E. P., Latrille, E., Souchon, I., \& Martin, N. (2003). Texture-flavor interactions in low fat stirred yogurt: How mechanical treatment, thickener concentration and aroma concentration affect perceived texture and flavor. Journal of Sensory Studies, 18, 367-390.

Li, Q., Chen, Q., Ruan, H., Zhu, D., \& He, G. (2010). Isolation and characterisation of an oxygen, acid and bile resistant Bifidobacterium animalis subsp. lactis Qq08. Journal of the Science of Food and Agriculture, 90, 1340-1346.

Lim, J. (2011). Hedonic scaling: A review of methods and theory. Food Quality and Preference, 22, 733-747.

MacFie, H. J., Bratchell, N., Greenhoff, K., \& Vallis, L. V. (1989). Designs to balance the effect of order of presentation and first-order carry-over effects in hall tests. Journal of Sensory Studies, 4, 129-148.

Majchrazak, D., Lahm, B., \& Durrschmid, K. (2010). Conventional and probiotic yogurts differ in sensory properties but not in consumers' preferences. Journal of Sensory Studies, 25, 431-446.

Marafon, A. P., Sumi, A., Alcântara, M. R., Tamime, A. Y., \& Oliveira, M. N. (2011). Optimization of the rheological properties of probiotic yoghurts supplemented with milk proteins. LWT-Food Science + Technology, 44, 511-519.

Martin, F., Cachon, R., Pernin, K., De Coninck, J., Gevais, P., Guichard, E., et al. (2011). Effect of oxidoreduction potential on aroma biosynthesis by lactic bacteria in nonfat yogurt. Journal of Dairy Science, 94, 614-622.

Menezes, E., Deliza, R., Cham, H. L., \& Guinard, J. -X. (2011). Preferences and attitudes towards açaí-based products among North American consumers. Food Research International, 44, 1997-2008.

Ngapo, T. M., Fortin, J., Aalhus, J. L., \& Martin, J. -F. (2010). Consumer choices of pork chops: Results from two Canadian sites. Food Research International, 43, 1559-1565.

Nunes, C. A., Pinheiro, A. C. M., \& Bastos, S. C. (2011). Evaluating consumer acceptance tests by three-way internal preference mapping obtained by parallel factor analysis. Journal of Sensory Studies, 26, 167-174.

Oliveira, R. P. S., Perego, P., Oliveira, M. N., \& Converti, A. (2011). Growth and survival of mixed probiotics in nonfat fermented milk: The effect of inulin. Chemical Engineering Transactions, 24, 457-462.

Ovejero-López, I., Bro, R., \& Bred, W. L. P. (2005). Univariate and multivariate modelling of flavour release in chewing gum using time-intensity: A comparison of data analytical methods. Food Quality and Preference, 16, 327-343.

Palazzo, A. B., \& Bolini, H. M. A. (2009). Multiple time-intensity analysis and acceptance of raspberry-flavored gelatin. Journal of Sensory Studies, 24, 648-663.

Saint-Eve, A., Kora, E. P., \& Martin, M. (2004). Impact of the olfactory quality and chemical complexity of the flavouring agent on the texture of low fat stirred yogurts assessed by three different sensory methodologies. Food Quality and Preference, $15,655-668$.

Tuorilla, H., \& Monteleone, E. (2009). Sensory science in the changing society: Opportunities, needs and challenges. Trends in Food Science and Technology, 20, 54-62.

Villanueva, N., \& Da Silva, M. A. A. (2009). Comparative performance of the nine-point hedonic, hybrid and self-adjusting scales in the generation of internal preference maps. Food Quality and Preference, 20, 1-12.

Villanueva, N. D. M., Doris, N., \& Trindade, M. A. (2010). Estimating sensory shelf life and carrot cupcakes using acceptance tests. Journal of Sensory Studies, 25, 260-279.

Yonezawa, S., Xiao, J. Z., Odamaki, T., Ishida, T., Miyaji, K., Yamada, A., Yaeshima, T., \& Iwatsuki, K. (2010). Improved growth of bifidobacteria by cocultivation with Lactococcus lactis subspecies lactis. Journal of Dairy Science, 83, 1815-1823. 